\title{
Trends in the incidence, treatment, and survival of patients with lung cancer in the last four decades
}

This article was published in the following Dove Medical Press journal: Cancer Management and Research

\author{
Tao Lu* \\ Xiaodong Yang* \\ Yiwei Huang \\ Mengnan Zhao \\ Ming Li \\ $\mathrm{Ke} \mathrm{Ma}$ \\ Jiacheng Yin \\ Cheng Zhan \\ Qun Wang
}

Department of Thoracic Surgery,

Zhongshan Hospital, Fudan University,

Shanghai, China

*These authors contributed equally to this work
Correspondence: Cheng Zhan;

Qun Wang

Department of Thoracic Surgery,

Zhongshan Hospital, Fudan University,

180 Fenglin Road, Shanghai 200032, China

Tel/fax +8621 64041990

Email czhan10@fudan.edu.cn;

wang.qun@zs-hospital.sh.cn
Purpose: This study used the Surveillance, Epidemiology, and End Results (SEER) data to investigate the changes in incidence, treatment, and survival of lung cancer from 1973 to 2015. Patients and methods: The clinical and epidemiological data of patients with lung cancer were obtained from the SEER database. Joinpoint regression models were used to estimate the rate changes in lung cancer related to incidence, treatment, and survival.

Results: From 1973 to 2015, the average incidence of lung cancer was 59.0/100,000 personyears. The incidence increased initially, reached a peak in 1992, and then gradually decreased. A higher incidence rate was observed in males than in females and in black patients than in other racial groups. Since 1985, adenocarcinoma became the most prevalent histopathological type. The surgical rate for lung cancer was about $25 \%$, and treatment with chemotherapy showed an increasing trend, while the radiotherapy rate was in downward trend. The surgical rate for non-small-cell lung cancer (NSCLC) was higher than that for small cell lung cancer (SCLC), while chemotherapy for SCLC far exceeded that for NSCLC. Treatment with chemotherapy and radiotherapy for advanced stage had higher rate than early stage. The 5-year relative survival rate of lung cancer increased with time, but $<21 \%$.

Conclusion: In the past four decades, the lung cancer incidence increased initially and then gradually decreased. Surgical rate experienced a fluctuant reduction, while the chemotherapy rate was in upward trend. The 5-year relative survival rate increased with years, but was still low.

Keywords: lung cancer, incidence, treatment, survival, SEER database

\section{Introduction}

Lung cancer is the leading cause of cancer death and is attributed to more than $25 \%$ of all cancer deaths. In the United States, it is estimated that $\sim 225,000$ patients are diagnosed with lung cancer with 160,000 deaths due to lung cancer annually. ${ }^{1}$ However, it has been reported that the incidence of lung cancer has significantly declined among men and women in all racial groups in recent decades. ${ }^{2,3}$

Primary lung cancer is typically divided into two main histopathological types: small cell lung cancer (SCLC) and non-small-cell lung cancer (NSCLC). NSCLC accounts for $85 \%$ of lung cancer cases, including three main subtypes, namely adenocarcinoma (ADC), squamous cell carcinoma (SCC), and large cell carcinoma (LCC). ${ }^{4}$ The trends in lung cancer incidence rates by histopathological types have shifted over the past few decades in the United States. ${ }^{5-8}$ Polednak ${ }^{9}$ found that the incidence rates of ADC, SCC, and SCLC decreased from 1992 to 2005 in the United States. This decline in incidence was also observed for LCC in another study..$^{10}$ During this period, 
the incidence of SCC was observed to decrease more rapidly than that of ADC. At present, $\mathrm{ADC}$ is more common than SCC in the United States. ${ }^{5,6}$

Lung cancer is one of the most aggressive malignant tumors, especially SCLC. The treatments for lung cancer mainly include surgical resection and chemotherapy. In general, patients with early-stage lung cancer usually undergo surgery, while patients with advanced or metastatic disease are treated with chemotherapy. ${ }^{11}$ However, treatment methods have also shifted over the decades. Despite improved treatment, lung cancer still has a poor prognosis and the 5-year survival rate remains at a very low level. ${ }^{12}$ Several factors, such as age, sex, lung function, clinical and pathological stage, body constitution, comorbidity, and optimal treatment, influence the survival of lung cancer patients. ${ }^{13}$

To our knowledge, there have been some publications on an international level to access the trend in some aspects of lung cancer, ${ }^{14,15}$ but no studies on the changes in incidence, treatment, and long-term survival rate of lung cancer spanning four decades have been carried out. In this study, we used the population-based Surveillance, Epidemiology and End Results (SEER) database, which represents $26 \%$ of the US population, to investigate changes in the incidence, treatment, and 5-year relative survival rate of lung cancer analyzed by sex, age, race, and histopathological type over time.

\section{Materials and methods}

The SEER database is an open access resource for cancerbased epidemiology and survival analyses. The SEER program includes 18 cancer registries collecting information on newly diagnosed cases within SEER geographic regions in the United States, and covers $\sim 97 \%$ of all incident cancers in its registry areas. More than 8 million cancer cases spanning four decades are registered in this database.

The data on patients with lung cancer were obtained from the SEER database via SEER*Stat, version 8.3.5 (http://seer.cancer.gov/seerstat/). We defined lung cancer using the ICD for Oncology, Version 3 (ICD-O-3). Patients with labeled primary sites, C34.1-upper lobe, lung, C34.2middle lobe, lung, C34.3-lower lobe, lung, and C34.8overlapping lesion of lung, C34.9-lung, not otherwise specified, were included in this study. Data on frequency and age were obtained from the database of Incidence-SEER 18 Research Data + Hurricane Katrina Impacted Louisiana Cases, November 2017 Sub, 1973-2015 varying. Data on the treatment are obtained from the database of IncidenceSEER 18 Regs Custom Data (with additional treatment fields), November 2017 Sub (1975-2015 varying). The surgical rate, radiotherapy rate, and chemotherapy rate were calculated manually. Data on incidence and survival rate were obtained from incidence 9 Regs Research Data, November 2017 Sub (1973-2015) <Katrina/Rita Population Adjustment $>$. The demographic and clinical data, including the year of diagnosis, age-adjusted incidence rates, age, sex, race/ethnicity, tumor stage, histopathologic types, treatment methods, and the 5-year relative rate, were used to estimate the changes in lung cancer over time. According to the SEER database, racial groups included white, black, other (American Indian/AK Native, Asian/Pacific Islander), and unknown racial patients. The data on unknown racial patients were omitted when calculating the incidence of different racial groups. The categories of lung cancer were defined by the 2015 WHO Classification of Lung Tumors. ${ }^{16}$ Histopathological types were defined using ICD-O-3 His/ behave, malignant. We calculated and analyzed the data of four histopathological types, namely ADC (8140/3, $8250 / 3,8251 / 3,8252 / 3,8253 / 3,8255 / 3,8260 / 3,8310 / 3)$, $\operatorname{SCC}(8070 / 3,8071 / 3,8072 / 3), \operatorname{LCC}(8012 / 3,8013 / 3)$, and SCLC $(8041 / 3,8044 / 3,8045 / 3)$. Tumor stage was classified as localized, regional, and distant according to SEER historical stage A, which contained data from 1988 to 2015. The TNM stage system was not adopted because the staging criteria were not consistent over the years. Incidence rates were age-adjusted using the 2000 United States Standard Population, and all incidence rates were reported as ageadjusted incidence per 100,000 person-years.

\section{Statistical analysis}

Joinpoint Regression Program, version 4.5.0.1 (Statistical Methodology and Applications Branch, Surveillance Research Program, National Cancer Institute) was used to estimate the time periods of significant increases or decreases in rates via Joinpoint regression models. The Joinpoint regression models tested which trends (between joinpoints) were statistically significant and then estimated the annual percentage change (APC) in rates between the two joinpoint years. A maximum of three joinpoints was applied to characterize trends. $P<0.05$ was considered significant and all tests were two-tailed.

\section{Ethical considerations}

Ethics approval was exempted by the Ethics Committee of Zhongshan Hospital of Fudan University (Shanghai, China), as the SEER is a publicly available database, and data extracted from SEER were identified as nonhuman study. All patient data were anonymized. 


\section{Results}

\section{Incidence of lung cancer}

According to the SEER database, 1,148,341 patients developed lung cancer from 1973 to 2015, including 646,662 males and 501,679 females. Of these, white patients comprised the majority ( $\mathrm{n}=960,808)$, followed by black patients $(n=122,079)$, and then other racial patients $(n=64,010)$, and unknown racial patients $(\mathrm{n}=1,444)$. A high incidence rate of lung cancer was observed with 59.0/100,000 person-years on average over the past 43 years. In 1973, the incidence of lung cancer was $47.5 / 100,000$ person-years. The overall incidence of lung cancer significantly increased from 1973 to 1991 , and reached a peak in $1992(65.9 / 100,000$ personyears). The incidence then decreased from 1991 to 2015, which was mainly due to a decrease in incidence in male patients. In 2015, the incidence dropped to $48.9 / 100,000$ person-years (Figure 1A).
Male patients showed a decreasing trend, while female patients showed an increasing trend in overall incidence in the past decades. The incidence rate in male patients increased from 1973 (83.5/100,000 person-years) to 1984 (97.9/100,000 person-years), and then gradually decreased to a historical low level of 55.3/100,000 person-years in 2015 . However, the incidence in female patients gradually increased from $20.2 / 100,000$ person-years in 1973 to $51.3 / 100,000$ person-years in 2007. The incidence subsequently decreased slowly and was 44.2/100,000 person-years in 2015. In general, the incidence in male patients was higher than that in female patients, but this gap has gradually diminished in recent years (Figure 1B). There was a significant change in the average age of patients with lung cancer from 1973 (64.1 years) to 2015 (70.3 years). With regard to racial groups, the incidence rate in black patients was the highest among all racial groups during the same time period (white vs black
A

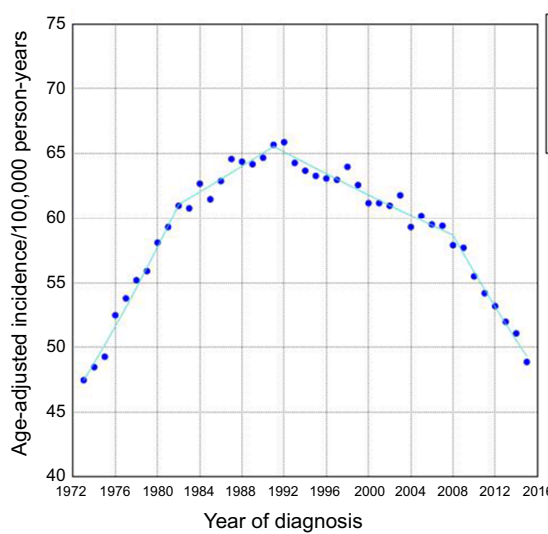

C

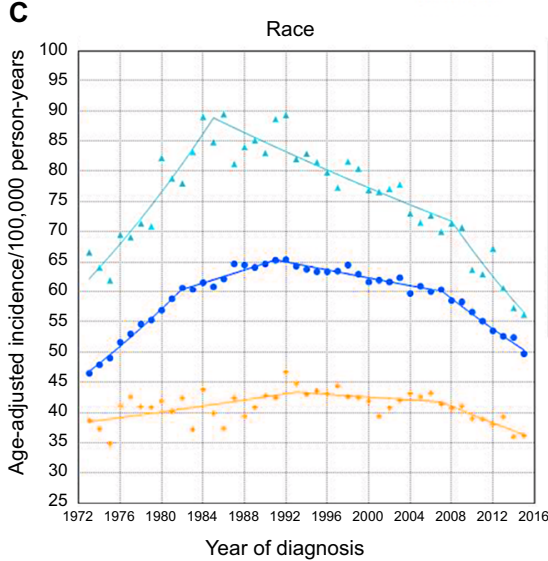

B
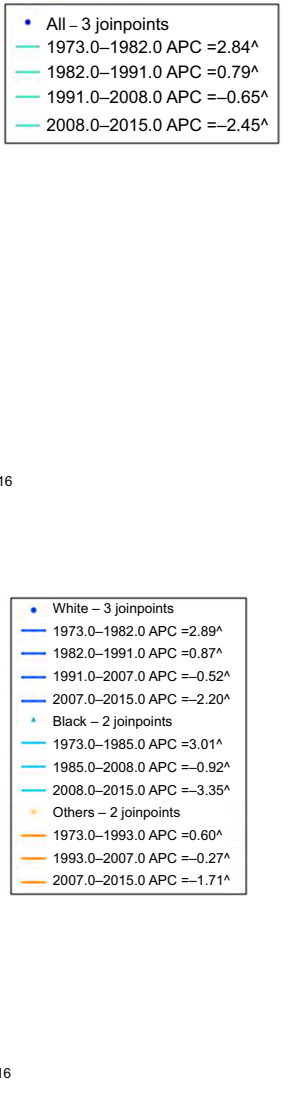

D

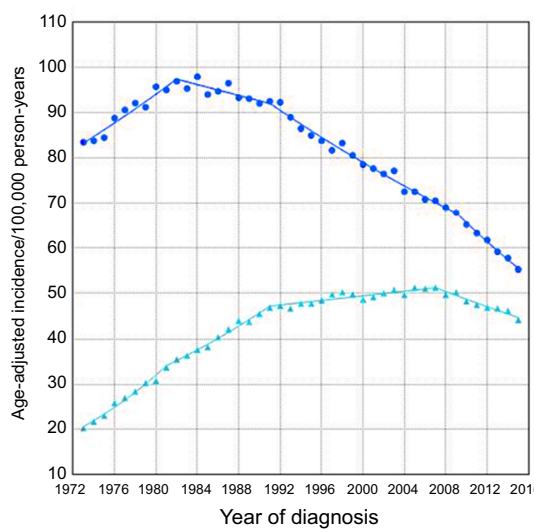

- Male -3 joinpoint

- 1973.0-1982.0 APC $=1.75^{\wedge}$

- 1982.0-1991.0 APC $=-0.63^{\wedge}$

- 1991.0-2009.0 APC $=-1.68^{\wedge}$

-2009.0-2015.0 APC $=-3.28^{1}$

Female -3 joinpoints

- 1973.0-1981.0 APC $=6.43^{\wedge}$

- 1981.0-1991.0 APC $=3.31^{\wedge}$

- 1991.0-2007.0 APC $=0.52^{\wedge}$ -2007.0-2015.0 APC $=-1.70^{\wedge}$

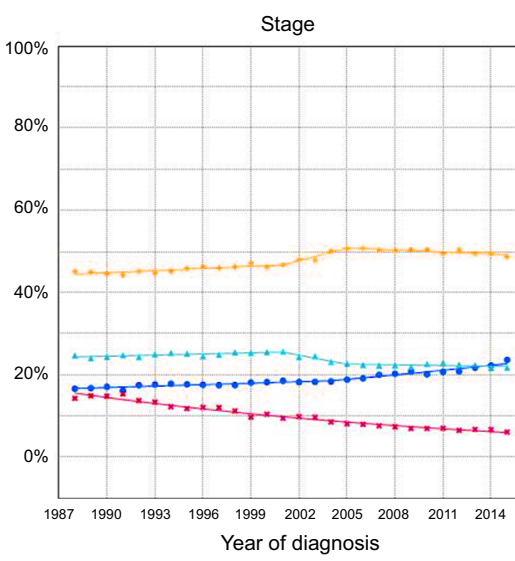

Figure I The change in incidence trends of lung cancer over years.

Notes: (A) The overall trend in the incidence of lung cancer from 1973 to 2015 . (B) The sex differences in the incidence of lung cancer. (C) The incidence of lung cancer in different racial groups. (D) The changes in tumor stages from 1988 to 2015 .

Abbreviation: APC, annual percentage change. 
vs others $=61.94$ vs 78.99 vs $42.39 / 100,000$ person-years on average). Black patients showed an increased incidence from 1973 to 1985 , and then a decrease from 1985 to 2015. In white patients, there was an increase in incidence from 1973 to 1991, followed by a significant decrease from 1991 to 2015 . The other racial groups showed the lowest incidence rates, which remained relatively stable from 1973 to 2015 (Figure 1C).

Lung cancer stage was divided into the following four categories: localized, regional, distant, and unstaged (Figure 1D). The average percentage of localized, regional, distant, and unstaged lung cancers was $18.8 \%, 23.7 \%, 47.7 \%$, and $10.0 \%$, respectively. Localized stage lung cancer showed an increasing trend, rising from $16.6 \%$ in 1988 to $23.6 \%$ in 2015. The percentage of regional stage lung cancer showed a slight decreasing trend (24.6\% in 1988 to $21.7 \%$ in 2015 ). The percentage of distant stage lung cancer far exceeded that of the other two stages, and remained at a high level with a general increasing trend $(45.2 \%$ in 1988 to $48.7 \%$ in 2015). For unstaged lung cancer, the percentage had been on a decline from 1988 (14.2\%) to 2015 (6.1\%).

The histopathological types of lung cancer mainly consisted of ADC, SCC, LCC, and SCLC (Figure 2). The average incidence rates of ADC, SCC, LCC, and SCLC were $17.9,13.3,3.1$, and $6.0 / 100,000$ person-years, respectively.
Overall, ADC showed an increasing trend in incidence from 1973 (8.9/100,000 person-years) to 2015 (20.3/100,000 person-years), while SCC showed a decreasing trend from $1973(13.3 / 100,000$ person-years) to $2015(9.8 / 100,000$ person-years). The incidence rate of SCC significantly increased from 1973 and reached a peak in 1984, and then decreased from 1984 to 2005 . Following a stable period, SCC showed a decreasing trend in incidence from 2011 to 2015. In general, the incidence rate of ADC increased from 1973 to 1992 . After a nonsignificant decrease, the incidence increased again from 2003 to 2015. In 1985, the incidence rate of ADC surpassed that of SCC for the first time and this disparity continuously increased. The incidence rate of LCC was relatively low, and increased initially (1973 to 1987) and then decreased (1987-2015). The incidence of SCLC was higher than that of LCC, and also showed periods of increased and decreased incidence. The incidence of lung cancer in male patients was higher than that in females for all four histopathological types, but the gap narrowed with time (Figure 3A-D). Notably, in females, the incidence of ADC significantly increased from $5.4 / 100,000$ person-years in 1973 to $19.5 / 100,000$ person-years in 2015 , whereas the incidence of SCC in male patients significantly decreased from $25.5 / 100,000$ person-years in 1973 to $13.3 / 100,000$ person-years in 2015.
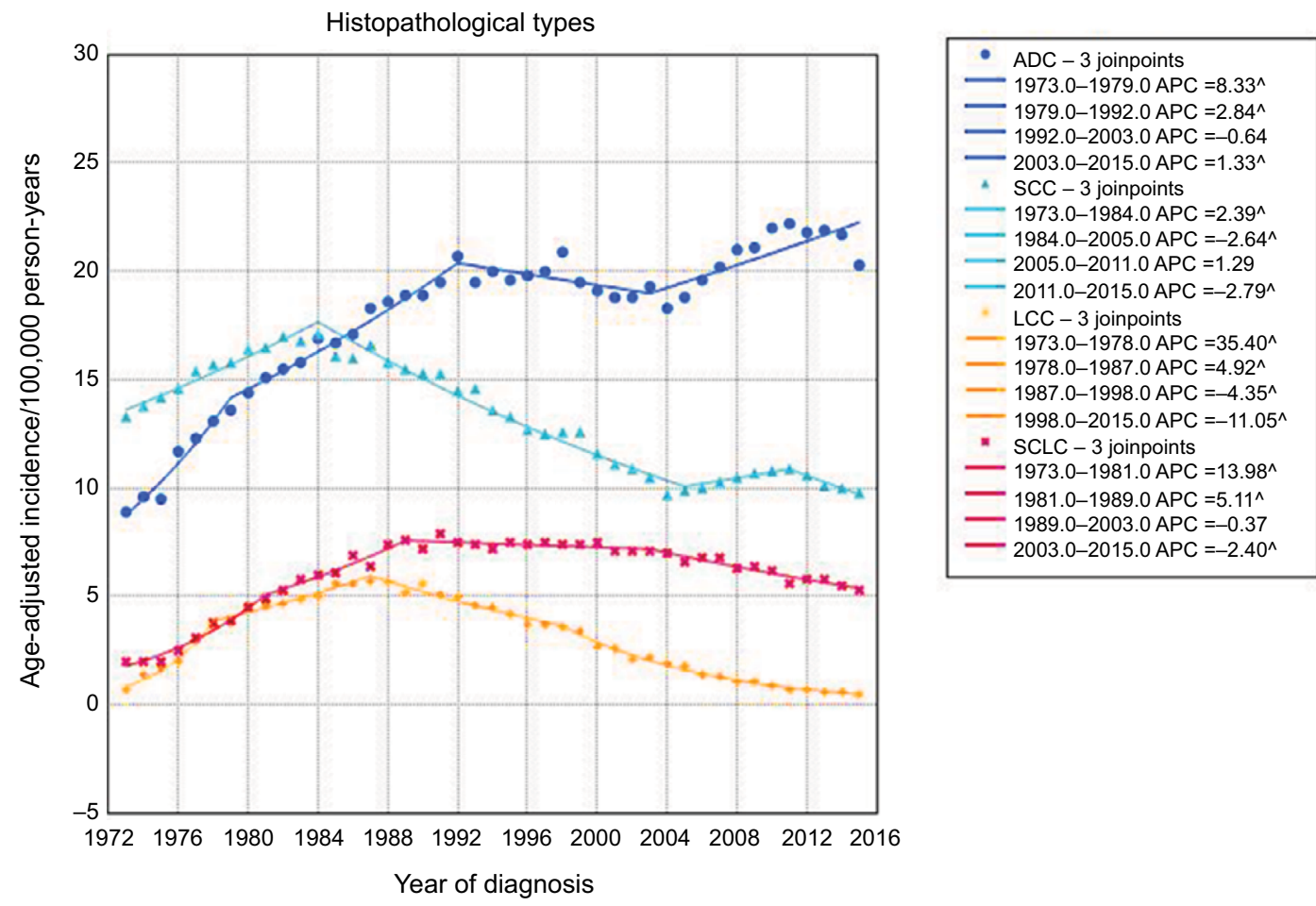

Figure 2 The trends in incidence of four main histopathological types of lung cancer from 1973 to 2015.

Abbreviations: ADC, adenocarcinoma; APC, annual percentage change; LCC, large cell carcinoma; SCC, squamous cell carcinoma; SCLC, small cell lung cancer. 

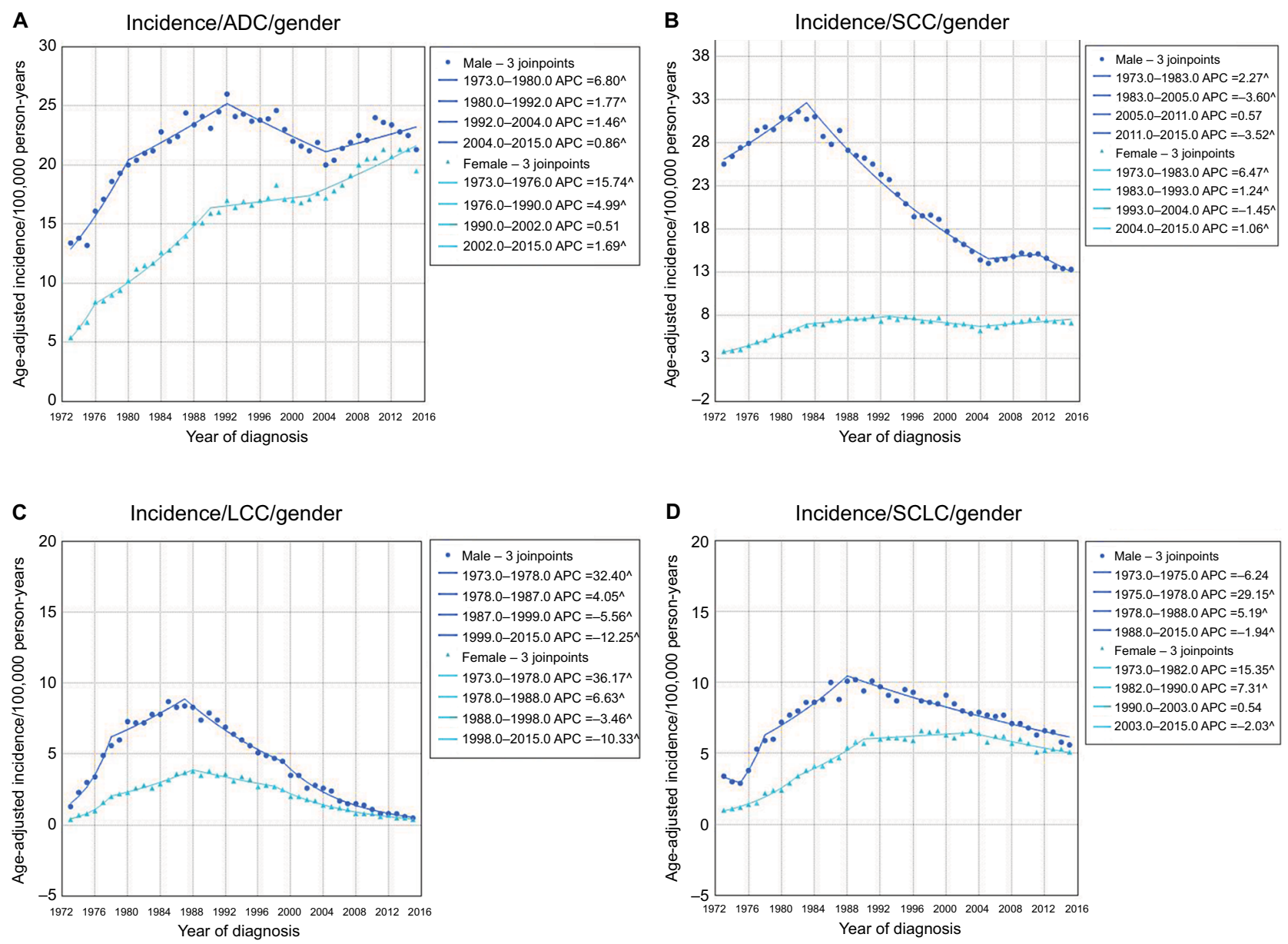

Figure 3 The sex differences in the incidence of ADC (A), SCC (B), LCC (C), and SCLC (D).

Abbreviations: ADC, adenocarcinoma; APC, annual percentage change; LCC, large cell carcinoma; SCC, squamous cell carcinoma; SCLC, small cell lung cancer.

\section{Treatment of lung cancer}

The main treatments for lung cancer include surgery, chemotherapy, and radiotherapy. In the present study, although the surgical rate showed slight fluctuations over time, it remained relatively stable at about $25 \%$. The radiotherapy rate experienced a general downward trend, but showed an increasing trend from 2007 (35.3\%) to 2015 (38.8\%). However, treatment with chemotherapy rose over the past decades, increasing from $14.9 \%$ in 1973 to $39.2 \%$ in 2015 (Figure 4).

The present study revealed that the surgical rates for NSCLC were much higher than those for SCLC (Figure 5A). From 1973 to 2015 , the highest average surgical rate was seen in ADC patients (36.6\%), followed by SCC (31.2\%), LCC (26.4\%), and SCLC patients (5.9\%). The surgical rates for ADC, SCC, and SCLC decreased over time, but the rate for LCC significantly increased from the late 1980s. As shown in Figure 5B, most patients with localized stage lung cancer underwent surgical treatment (an average of 61.6\%). However, the surgical rate significantly decreased from 1988 to
2015. The average surgical rate for regional stage lung cancer was $38.2 \%$, ranking second, while only $5.1 \%$ of patients with distant stage lung cancer underwent surgery, and this rate declined from 1988 to 2015. For unstaged tumor, the average surgical rate was $3.6 \%$, and showed a decreased trend from 1973 to 2015.

All four histopathological types of lung cancer showed an increasing trend in treatment with chemotherapy from 1973 to 2015 (Figure 5C). In 1973, the rates of chemotherapy for ADC, SCC, LCC, and SCLC were 19.6\%, 11.7\%, 17.1\%, and $24.2 \%$, respectively. Following a continuous increase in chemotherapy, the rates of chemotherapy in these four histopathological types rose to $43.0 \%, 39.2 \%, 50.2 \%$, and $67.8 \%$, respectively. Notably, on average $65.4 \%$ of SCLC patients received chemotherapy, which was much higher than that in the other three histopathological types. However, the rate of chemotherapy in SCLC patients decreased from 1987 to 2015. For regional and distant stage lung cancer, the rate of chemotherapy increased from 1988 to 2015. In 2015, the 

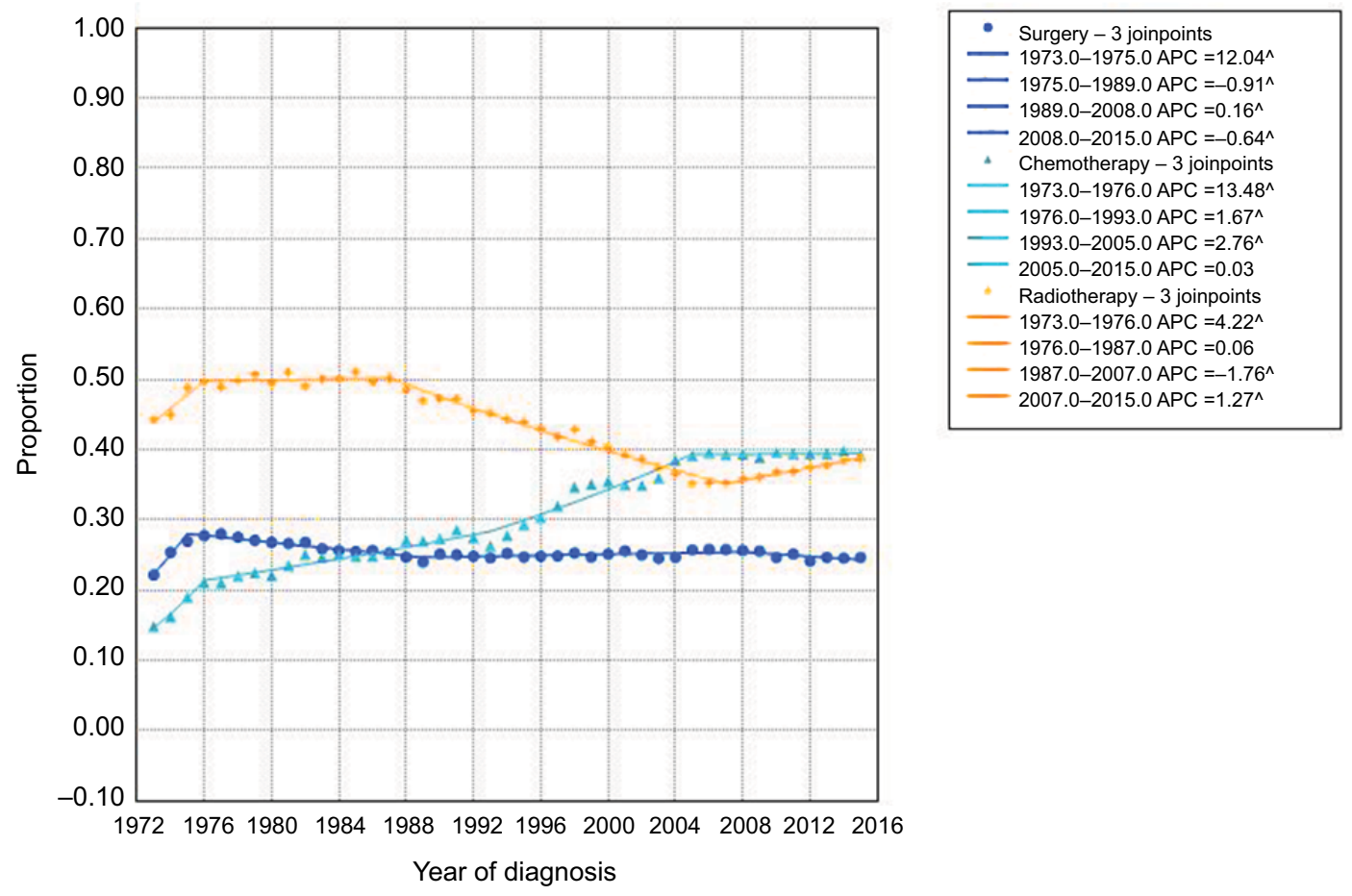

Figure 4 The rates of surgery, chemotherapy, and radiotherapy for lung cancer over the years.

Abbreviation: APC, annual percentage change.

rates of chemotherapy for regional and distant stage lung cancer were $53.3 \%$ and $51.2 \%$, respectively, while on average, only $12.4 \%$ of patients with localized stage lung cancer were treated with chemotherapy (Figure 5D). The treatment with radiotherapy for NSCLC experienced a significantly decreasing trend from 1980s to 2006. Since then, NSCLC have an increasing trend in radiotherapy rate. But for SCLC, the radiotherapy rate increased initially, and then rapidly went to the bottom in 1989 (38.7\%). Later, it increased gradually, reaching $44.5 \%$ in 2015 (Figure 5E). Regional and distant stage lung cancer had higher radiotherapy rate than localized stage. The radiotherapy rate of both regional and distant stage lung cancer experienced a decreasing trend from 1988 to the mid 2000s, and then remained stable (Figure 5F).

\section{Long-term survival of lung cancer patients}

In 1973, the 5-year relative survival rate of all patients with lung cancer was only $10.7 \%$ (Figure 6A). Although the survival rate has increased over the past four decades, it is still poor $(<21.0 \%)$. In 2010, the 5-year relative survival rate increased to $19.8 \%$.

Female patients had a better survival rate than male patients at all time points (Figure 6B). The average survival rate of male patients was $13.0 \%$, while the rate in female patients was $17.7 \%$. All racial groups showed an increasing trend in survival rate over the years. Black patients had the lowest survival rate at all time points, while white patients and other racial patients had better survival rates (Figure 6C). In general, aged lung cancer patients had a poorer survival rate than younger patients (Figure 6D). Notably, all age groups showed a marked increase in survival rate, especially patients aged 15-44 years.

Overall, the survival rate in patients with all four histopathological types of lung cancer increased (Figure 6E). The average 5-year survival rates for ADC, SCC, LCC, and SCLC from 1973 to 2010 were 20.6\%, 17.6\%, 13.2\%, and $5.6 \%$, respectively. Compared with the survival rate in 1973, the rates for ADC, SCC, LCC, and SCLC increased by $10.5 \%, 7.8 \%, 10.3 \%$, and $2.8 \%$ over the past decades, reaching $26.2 \%, 21.3 \%, 21.1 \%$, and $7.2 \%$, respectively, in 2010. Patients with NSCLC had a much better survival rate than those with SCLC. On the whole, patients with localized, regional, and distant stage lung cancer had an increase in 5-year relative survival rate from 1988 to 2010 , respectively. Patients with localized stage lung cancer had the highest survival rate from 1988 (45.3\%) to 2010 (58.2\%), with an average of $50.3 \%$. The survival rate for regional stage lung cancer was $22.2 \%$ on average. Although the survival rate for distant stage lung cancer increased from 1988 to 2010, 
A
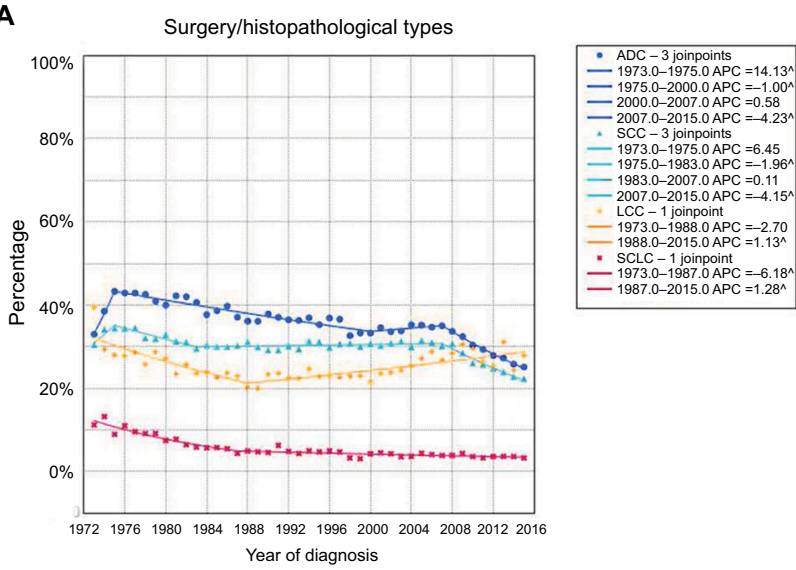

C
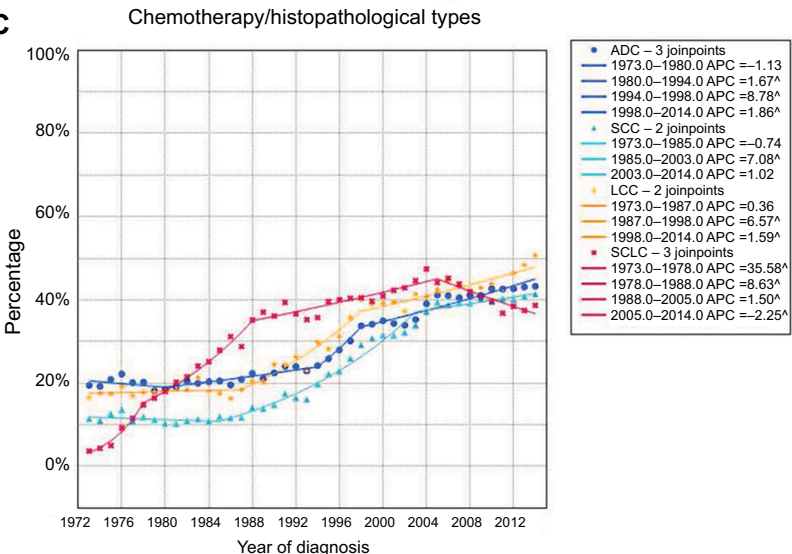

E
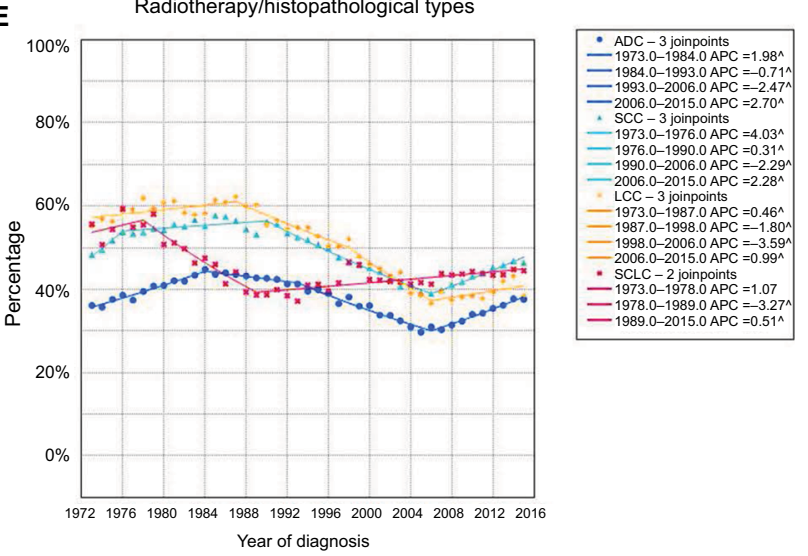

B
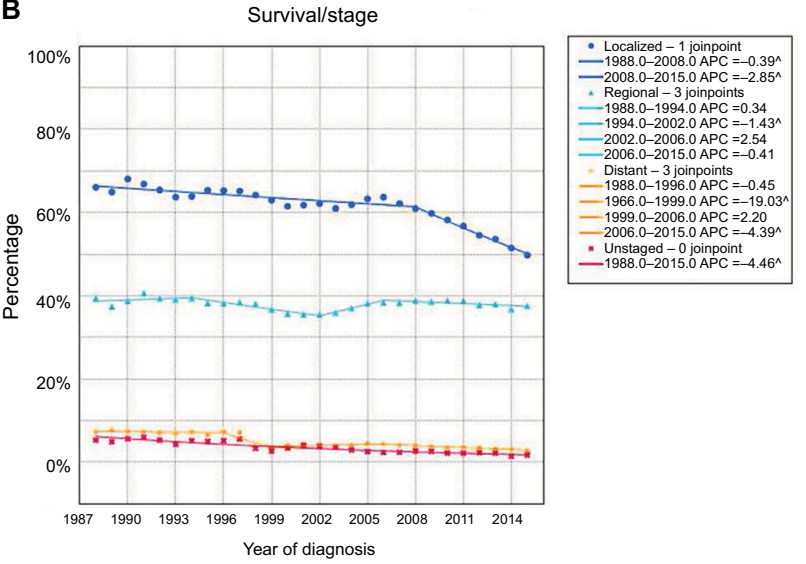

D
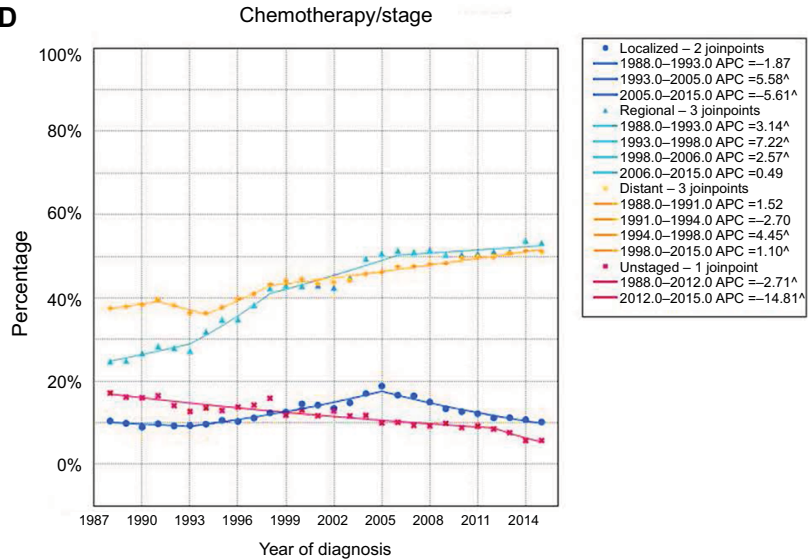

Figure $\mathbf{5}$ The rates of different treatments for lung cancer over years.

$\mathbf{F}$
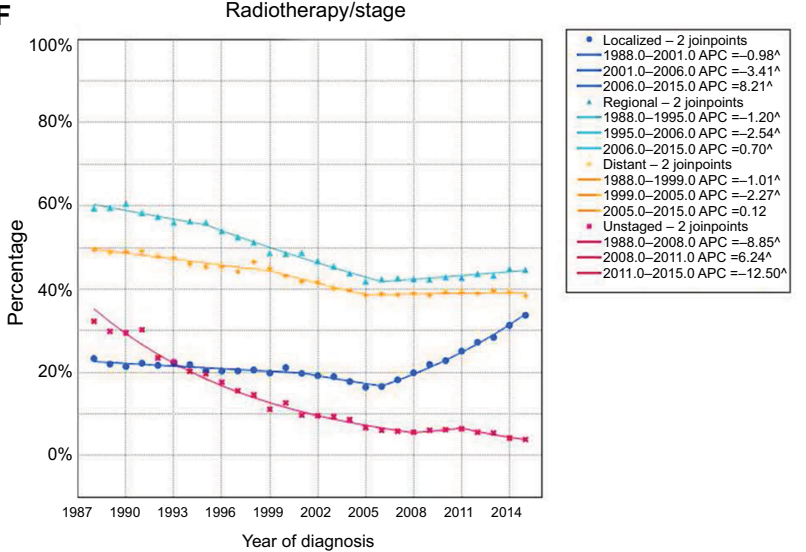

Notes: (A) The surgical rates for different histopathological types of lung cancer. (B) The surgical rates for different stages of lung cancer. (C) The rate of chemotherapy for different histopathological types of lung cancer. (D) The rate of chemotherapy for different stages of lung cancer. (E) The rate of radiotherapy for different histopathological types of lung cancer. (F) The rate of radiotherapy for different stages of lung cancer.

Abbreviations: ADC, adenocarcinoma; APC, annual percentage change; LCC, large cell carcinoma; SCC, squamous cell carcinoma; SCLC, small cell lung cancer.

only $2.9 \%$ of patients on average survived more than 5 years (Figure 6F).

\section{Discussion}

In the present study, we used Joinpoint regression models to estimate the APC change in trends in lung cancer incidence and prevalence by age, sex, race, and histopathological types, and calculated the proportion of tumors in different stages, various treatments, and the long-term survival of patients with lung cancer.

The present study showed that lung cancer incidence has declined in the past 20 years, which is thought to be 
A

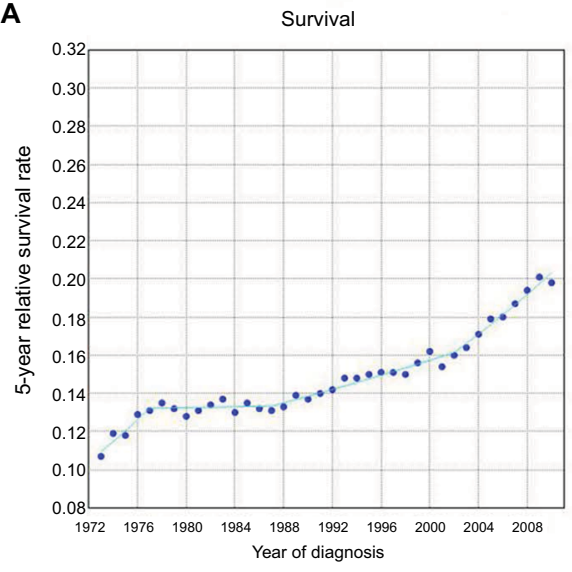

C

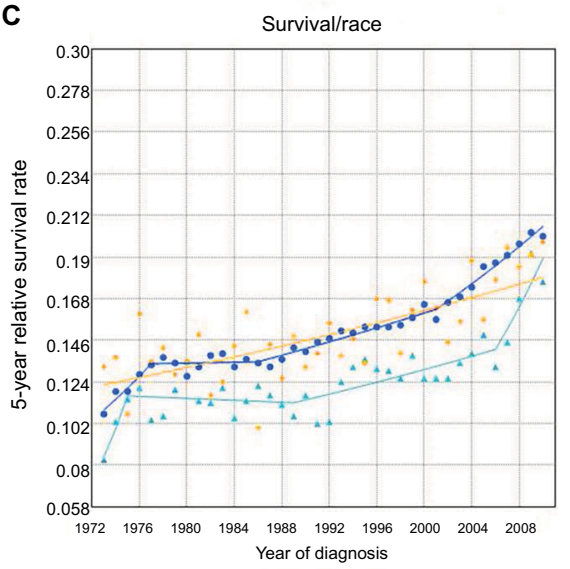

E

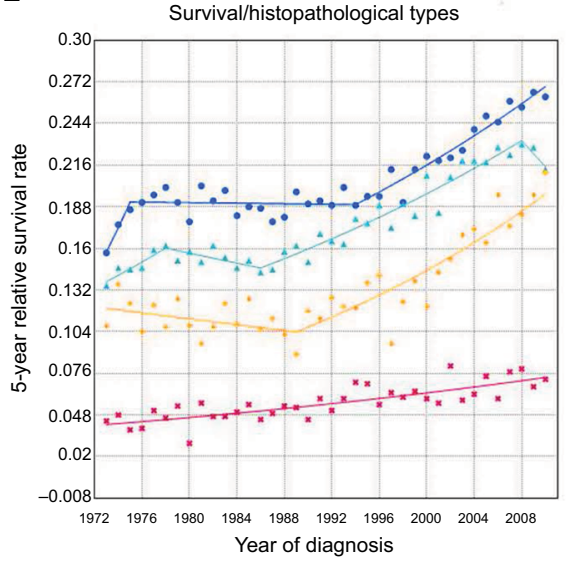

B

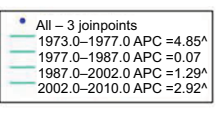

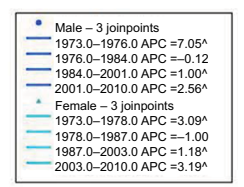

Survival/gender

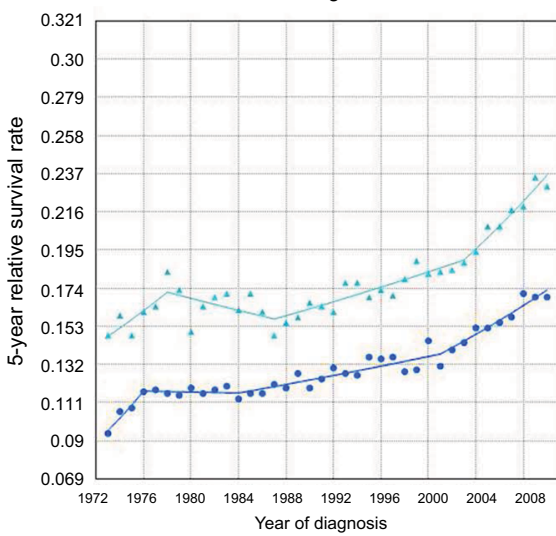

D

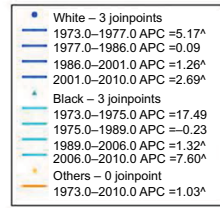

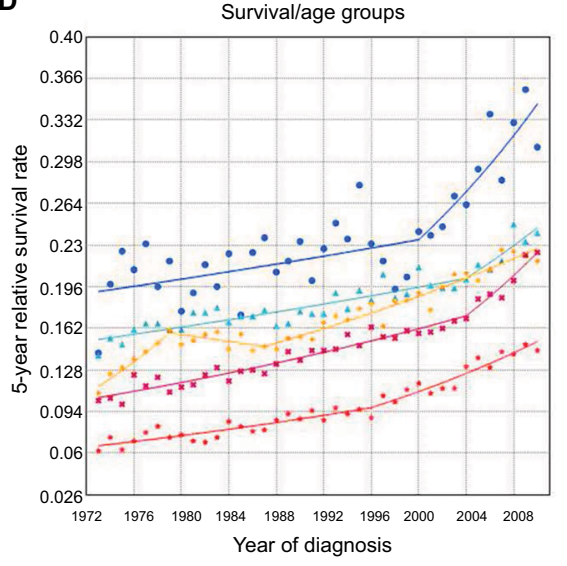

$\mathbf{F}$

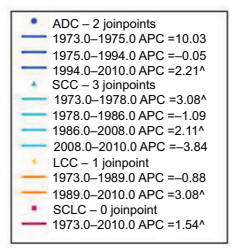

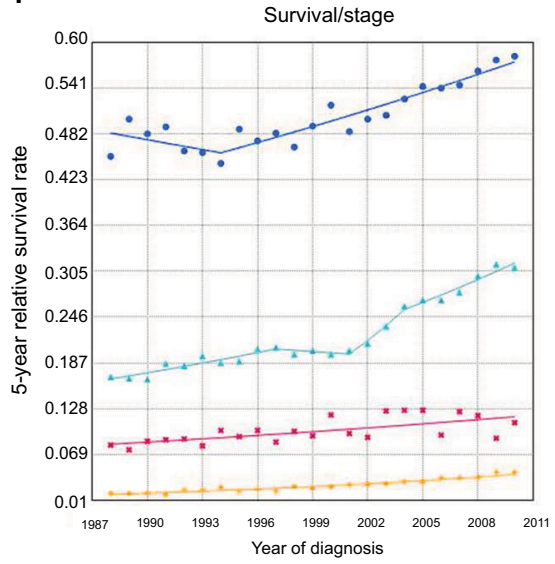

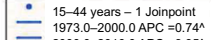
$\div$ 2000.0-2010.0 APC $=3.95 n$

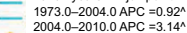

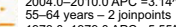
\begin{aligned} $1973.0-1979.0 \mathrm{APC}=5.55^{1} \\ -1979.0-1987.0 \mathrm{APC}=-0.91 \\$\hline\end{aligned} - $1987.0-2010.0 \mathrm{APC}=1.911$

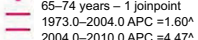
- 75 + years -1 joinpoint

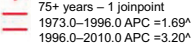

Figure 6 The long-term survival of lung cancer patients.

Notes: (A) The 5-year relative survival rate of lung cancer from 1973 to 2010 . The 5-year relative survival of lung cancer patients by sex (B), race (C), age group (D), histopathological type (E), and different stage (F).

Abbreviations: ADC, adenocarcinoma; APC, annual percentage change; LCC, large cell carcinoma; SCC, squamous cell carcinoma; SCLC, small cell lung cancer.

largely due to tobacco control programs. ${ }^{2,9}$ Nonetheless, this decline is not consistent among men and women and all racial groups, thus causing disparities in lung cancer incidence. Men appeared to be more susceptible to lung cancer than women historically. $\mathrm{Hu}$ et $\mathrm{al}^{17}$ reported that cigarette smoking in both sexes has significantly declined, but men had higher smoking rates than women at any time point, which was reflected in the higher lung cancer incidence rates in men. However, our study showed that the decline in men was sharper, and the gender gap in lung cancer incidence has 
narrowed in recent years. Moreover, a recent study reported that the historically higher incidence rates in young men compared with young women have reversed since 1960s, which are not fully explained by sex differences in smoking behavior. ${ }^{18}$ Therefore, future studies are needed to identify the reasons for the higher incidence of lung cancer in young women. Many reports have shown that the incidence rates of lung cancer in black individuals are higher than those in white individuals, ${ }^{19-21}$ although this racial gap also narrowed over time, which is consistent with our findings. The higher incidence rates in black individuals may be due to multiple factors, such as a greater susceptibility to smoking, ${ }^{22}$ poorer access to health care services, ${ }^{23}$ and lower smoking cessation rates. ${ }^{24}$ As effective early screening methods for lung cancer are lacking, and clinical symptoms do not appear until the disease has progressed, the majority of patients are diagnosed with lung cancer at advanced stages. ${ }^{25}$

It is well-known that lung cancer etiology and prognosis are correlated with tumor histopathological types. The trends in lung cancer incidence vary greatly by histopathological type due to different exposure to tobacco smoke and other lung carcinogens. ${ }^{26-29}$ In our study, up to the early 1980 s, SCC was most prevalent in the United States, and then started to decrease, which paralleled a decline in smoking rates. ${ }^{6,8}$ It is considered that SCC oncogenesis is closely correlated with heavy smoking. ${ }^{30-32}$ In 1985, the incidence of ADC surpassed that of SCC for the first time, and ADC then became the most prevalent histopathological type of lung cancer. Although the smoking rate has declined, there are other risk factors associated with the increasing rate of ADC, such as human papillomavirus infection ${ }^{33}$ and both indoor and outdoor air pollution. ${ }^{34}$ In our study, the incidence of ADC showed an upward trend, while the incidence of other histological types (SCC, LCC, and SCLC), which are strongly associated with smoking, decreased from the late 1980s.

We showed that the 5-year relative survival rate for lung cancer significantly increased with time in the United States, which was mainly due to progress in treatment over the years. ${ }^{35}$ However, the survival of lung cancer patients is still poor. A study from Japan reviewed that the 5-year relative survival between $1970 \mathrm{~s}$ and $2000 \mathrm{~s}$ increased from $6.0 \%$ to $30.9 \%$ in females and from $7.2 \%$ to $19.6 \%$ in males. ${ }^{36}$ It is well-known that surgery offers lung cancer patients the best chance of long-term survival; however, our study revealed that the surgical resection rate was only about $25 \%$, and showed a decreasing trend in recent years. Similar to our results, a study from Norway showed that the surgical rate for lung cancer was $<20 \%$. From the mid 1990 s to the mid 2000 s, the lung cancer resection rate (including SCLC) increased from $16.4 \%$ to $19.1 \%$. Excluding SCLC cases, the resection rate increased from $19.7 \%$ to $20.2 \% .{ }^{37}$ In a study from Canada, only $18 \%$ of NSCLC received surgical treatment. For early-stage lung cancer, the surgical resection rate was $58.9 \%,{ }^{38}$ which was consistent with ours. We showed that the surgical rate has significantly decreased in localized lung cancer recently. It seems that the reason is that stereotactic radiotherapy has increased. ${ }^{39}$ In our study, the radiotherapy rate of localized lung cancer has an increasing trend from 2006 to 2015, which may explain why the surgical rate and chemotherapy rate decreased in this period. Moreover, most patients were diagnosed with distant stage lung cancer, which is another important risk factor for poor survival. Notably, female patients had better survival than male patients. This survival advantage is likely due to a variety of factors. Fu et $\mathrm{al}^{40}$ analyzed the SEER database and found that female patients with local stage lung cancer received surgery more frequently than males. This difference in surgical rate may partially explain the better survival rate in women. In addition, women were more vulnerable to ADC due to the difference in smoking patterns. ${ }^{29}$ During the 21 st century, ADC had a better prognosis than other histological types. ${ }^{36}$ The present study showed that the survival rates of black patients were lower than those of white patients at all time points, which may be attributed to the differences in tumor stage at diagnosis, variability in treatment, access to health care services, and socioeconomic factors. ${ }^{41,42}$ In addition, it has been reported that compared with white patients, black patients were less likely to undergo surgery for early-stage lung cancer or chemoradiotherapy for advanced stage lung cancer. ${ }^{25}$ Furthermore, black patients were more likely to be diagnosed with advanced lung cancer than white patients. These factors may contribute to the racial disparities observed in lung cancer survival in the United States.

\section{Limitations}

The SEER database only contains data on patients within US regions, and does not represent changes in the incidence, treatment, and survival of lung cancer worldwide. Therefore, extensive research is necessary to estimate the changes in lung cancer over the past decades.

\section{Conclusion}

This study updated the trends in lung cancer incidence by sex, race, age, and histopathological types, and showed changes in treatment and the 5-year relative survival. In the past decades, the overall incidence of lung cancer increased initially and 
then gradually decreased. The surgical rate and radiotherapy rate of lung cancer showed a general downward trend while the chemotherapy rate experienced a significantly increasing trend. Although the 5-year relative survival rate increased significantly with years, it was still very low. Our study provided a comprehensive overview of lung cancer over the past 43 years.

\section{Acknowledgments}

This work was supported by the National Natural Science Foundation of China (Grant No. 81472225; www.nsfc.gov. $\mathrm{cn} /$ ), and the Research Program of Shanghai Health and Family Planning Commission (201640102; www.wsjsw. gov.cn). The authors thank International Science Editing (http://www.internationalscienceediting.com) for editing this manuscript.

\section{Author contributions}

TL, XY, YH, MZ, ML, KM, JY, CZ, and QW conceived the study design. Data acquisition was carried out by TL, XY, YH, MZ, ML, KM, JY, and CZ. TL, XY, YH, and MZ did the analyses and interpretation of data. TL and XY drafted the manuscript. Revision of the manuscript was critically done by $\mathrm{CZ}$ and QW. All authors contributed to data analysis, drafting and revising the article, gave final approval of the version to be published, and agree to be accountable for all aspects of the work.

\section{Disclosure}

The authors report no conflicts of interest in this work.

\section{References}

1. Siegel RL, Miller KD, Jemal A. Cancer statistics, 2016. CA Cancer J Clin. 2016;66(1):7-30.

2. Mowls DS, McCaffree DR, Beebe LA. Trends in lung cancer incidence rates, Oklahoma 2005-2010. PLoS One. 2015;10(4):e0119251.

3. Jemal A, Ward EM, Johnson CJ, et al. Annual report to the nation on the status of cancer, 1975-2014, featuring survival. J Natl Cancer Inst. 2017;109(9).

4. Subramanian J, Regenbogen T, Nagaraj G, et al. Review of ongoing clinical trials in non-small-cell lung cancer: a status report for 2012 from the ClinicalTrials.gov Web site. J Thorac Oncol. 2013;8(7): 860-865.

5. Vincent RG, Pickren JW, Lane WW, et al. The changing histopathology of lung cancer: a review of 1682 cases. Cancer. 1977;39(4):1647-1655.

6. Devesa SS, Shaw GL, Blot WJ. Changing patterns of lung cancer incidence by histological type. Cancer Epidemiol Biomarkers Prev. 1991;1(1):29-34.

7. Govindan R, Page N, Morgensztern D, et al. Changing epidemiology of small-cell lung cancer in the United States over the last 30 years: analysis of the surveillance, epidemiologic, and end results database. J Clin Oncol. 2006;24(28):4539-4544.

8. Travis WD, Lubin J, Ries L, Devesa S. United States lung carcinoma incidence trends: declining for most histologic types among males, increasing among females. Cancer. 1996;77(12):2464-2470.
9. Polednak AP. Lung cancer incidence trends by histologic type in areas of California vs. other areas in the surveillance, epidemiology and end results program. Cancer Epidemiol. 2009;33(5):319-324.

10. Noone AM, Cronin KA, Altekruse SF, et al. Cancer incidence and survival trends by subtype using data from the surveillance epidemiology and end results program, 1992-2013. Cancer Epidemiol Biomarkers Prev. 2017;26(4):632-641.

11. Islam KM, Jiang X, Anggondowati T, Lin G, Ganti AK. Comorbidity and survival in lung cancer patients. Cancer Epidemiol Biomarkers Prev. 2015;24(7):1079-1085.

12. Thomas A, Chen Y, Yu T, Jakopovic M, Giaccone G. Trends and characteristics of young non-small cell lung cancer patients in the United States. Front Oncol. 2015;5:113.

13. Dela Cruz CS, Tanoue LT, Matthay RA. Lung cancer: epidemiology, etiology, and prevention. Clin Chest Med. 2011;32(4):605-644.

14. Lortet-Tieulent J, Soerjomataram I, Ferlay J, Rutherford M, Weiderpass $\mathrm{E}$, Bray F. International trends in lung cancer incidence by histological subtype: adenocarcinoma stabilizing in men but still increasing in women. Lung Cancer. 2014;84(1):13-22.

15. Varlotto JM, Voland R, McKie K, et al. Population-based differences in the outcome and presentation of lung cancer patients based upon racial, histologic, and economic factors in all lung patients and those with metastatic disease. Cancer Med. 2018;7(4):1211-1220.

16. Travis WD, Brambilla E, Nicholson AG, et al. The 2015 World health organization classification of lung tumors: impact of genetic, clinical and radiologic advances since the 2004 classification. J Thorac Oncol. 2015;10(9):1243-1260.

17. Hu SS, Neff L, Agaku IT, et al. Tobacco product use among adults - United States, 2013-2014. MMWR Morb Mortal Wkly Rep. 2016;65(27):685-691.

18. Jemal A, Miller KD, Ma J, et al. Higher lung cancer incidence in young women than young men in the United States. $N$ Engl $J$ Med. 2018;378(21):1999-2009.

19. Liff JM, Chow WH, Greenberg RS. Rural-urban differences in stage at diagnosis. Possible relationship to cancer screening. Cancer. 1991;67(5):1454-1459.

20. Bach PB, Cramer LD, Warren JL, Begg CB. Racial differences in the treatment of early-stage lung cancer. N Engl J Med. 1999;341(16):1198-1205.

21. Paquette I, Finlayson SR. Rural versus urban colorectal and lung cancer patients: differences in stage at presentation. J Am Coll Surg. 2007;205(5):636-641.

22. Haiman CA, Stram DO, Wilkens LR, et al. Ethnic and racial differences in the smoking-related risk of lung cancer. $N$ Engl $J$ Med. 2006;354(4):333-342.

23. Nelson AR. Unequal treatment: report of the Institute of Medicine on racial and ethnic disparities in healthcare. Ann Thorac Surg. 2003;76(4):S1377-S1381.

24. Gilpin EA, Pierce JP. Demographic differences in patterns in the incidence of smoking cessation: United States 1950-1990. Ann Epidemiol. 2002;12(3):141-150.

25. Hardy D, Liu CC, Xia R, et al. Racial disparities and treatment trends in a large cohort of elderly black and white patients with nonsmall cell lung cancer. Cancer. 2009;115(10):2199-2211.

26. Janssen-Heijnen ML, Coebergh JW. The changing epidemiology of lung cancer in Europe. Lung Cancer. 2003;41(3):245-258.

27. Au JS, Mang OW, Foo W, Law SC. Time trends of lung cancer incidence by histologic types and smoking prevalence in Hong Kong 1983-2000. Lung Cancer. 2004;45(2):143-152.

28. Toyoda Y, Nakayama T, Ioka A, Tsukuma H. Trends in lung cancer incidence by histological type in Osaka, Japan. Jpn J Clin Oncol. 2008;38(8):534-539.

29. Devesa SS, Bray F, Vizcaino AP, Parkin DM. International lung cancer trends by histologic type: male:female differences diminishing and adenocarcinoma rates rising. Int J Cancer. 2005;117(2):294-299.

30. Pesch B, Kendzia B, Gustavsson P, et al. Cigarette smoking and lung cancer - relative risk estimates for the major histological types from a pooled analysis of case-control studies. Int $J$ Cancer. 2012;131(5):1210-1219. 
31. Hinds MW, Stemmermann GN, Yang HY, Kolonel LN, Lee J, Wegner E. Differences in lung cancer risk from smoking among Japanese, Chinese and Hawaiian women in Hawaii. Int J Cancer. 1981;27(3):297-302.

32. Harris RE, Zang EA, Anderson JI, Wynder EL. Race and sex differences in lung cancer risk associated with cigarette smoking. Int J Epidemiol. 1993;22(4):592-599.

33. Li YJ, Tsai YC, Chen YC, Christiani DC. Human papilloma virus and female lung adenocarcinoma. Semin Oncol. 2009;36(6):542-552.

34. Chen F, Cole P, Bina WF. Time trend and geographic patterns of lung adenocarcinoma in the United States, 1973-2002. Cancer Epidemiol Biomarkers Prev. 2007;16(12):2724-2729.

35. Finks JF, Osborne NH, Birkmeyer JD. Trends in hospital volume and operative mortality for high-risk surgery. $N$ Engl J Med. 2011;364(22):2128-2137.

36. Kinoshita FL, Ito Y, Morishima T, Miyashiro I, Nakayama T. Sex differences in lung cancer survival: long-term trends using population-based cancer registry data in Osaka, Japan. Jpn J Clin Oncol. 2017;47(9):863-869.
37. Strand TE, Bartnes K, Rostad H. National trends in lung cancer surgery Eur J Cardiothorac Surg. 2012;42(2):355-358.

38. Bendzsak AM, Waddell TK, Urbach DR, Darling GE. Surgery and surgical consult rates for early stage lung cancer in Ontario: a populationbased study. Ann Thorac Surg. 2017;103(3):906-910.

39. Li M, Yang X, Chen Y, et al. Stereotactic body radiotherapy or stereotactic ablative radiotherapy versus surgery for patients with T1-3N0M0 non-small cell lung cancer: a systematic review and meta-analysis. Onco Targets Ther. 2017;10:2885-2892.

40. Fu JB, Kau TY, Severson RK, Kalemkerian GP. Lung cancer in women: analysis of the national surveillance, epidemiology, and end results database. Chest. 2005;127(3):768-777.

41. Coughlin SS, Matthews-Juarez P, Juarez PD, Melton CE, King M. Opportunities to address lung cancer disparities among African Americans. Cancer Med. 2014;3(6):1467-1476.

42. O'Keefe EB, Meltzer JP, Bethea TN. Health disparities and cancer: racial disparities in cancer mortality in the United States, 2000-2010. Front Public Health. 2015;3:51.
Cancer Management and Research

\section{Publish your work in this journal}

Cancer Management and Research is an international, peer-reviewed open access journal focusing on cancer research and the optimal use of preventative and integrated treatment interventions to achieve improved outcomes, enhanced survival and quality of life for the cancer patient. The manuscript management system is completely online and includes

\section{Dovepress}

a very quick and fair peer-review system, which is all easy to use. Visit $\mathrm{http}: / /$ www.dovepress.com/testimonials.php to read real quotes from published authors. 